European Conference on Laboratory Astrophysics - ECLA

C. Stehlé, C. Joblin and L. d'Hendecourt (eds)

EAS Publications Series, 58 (2012) 167-171

www.eas.org

\title{
NUMERICAL SIMULATIONS OF Z-PINCH EXPERIMENTS TO CREATE SUPERSONIC DIFFERENTIALLY-ROTATING PLASMA FLOWS
}

\author{
M. Bocchi ${ }^{1}$, B. Ummels ${ }^{1}$, J.P. Chittenden ${ }^{1}$ and S.V. Lebedev ${ }^{1}$
}

\begin{abstract}
In the context of high energy density laboratory astrophysics, we aim to produce and study a rotating plasma relevant to accretion discs physics. We devised an experimental setup based on a modified cylindrical wire array and we studied it numerically with the three-dimensional, resistive magneto-hydrodynamic code GORGON. The simulations show that a rotating plasma cylinder is formed, with typical rotation velocity $\sim 35 \mathrm{~km} / \mathrm{s}$ and Mach number $\sim 5$. In addition, the plasma ring is differentially rotating and strongly radiatively cooled. The introduction of external magnetic fields is discussed.
\end{abstract}

\section{Introduction}

Accretion discs are tightly linked to the formation of astrophysical jets, powerful outflows emanating from a variety of astronomical objects: from protostars to Active Galactic Nuclei (AGN) and quasars. The physics of accretion discs, however, is not completely understood. Accretion itself on the central object requires a high level of anomalous viscosity, which cannot be achieved by ordinary particle viscosity (Shakura \& Sunyaev 1973). The Magneto-Rotational Instability (MRI) is usually invoked to provide the required viscosity (Balbus \& Hawley 1991).

Laboratory experiments relevant to accretion discs have focused on the MRI employing Taylor-Couette flows. However, plasma experiments would allow a range of dimensionless parameters much closer to the astrophysical case (Ji 2011). Ryutov (2011) recently proposed a laser experiment which can potentially be used to study the level of viscosity in the discs. In this work we propose complementary experimental setups that employ a pulsed power machine instead. A twisted conical wire array configuration was previously used to produce rotating plasma jets on the MAGPIE pulsed power generator (Ampleford 2008). In this work, we

${ }^{1}$ Imperial College, Blackett Laboratory, SW7 2BW London, UK

(C) The Author(s) 2013

DOI: $10.1051 /$ eas $/ 1258027$ 


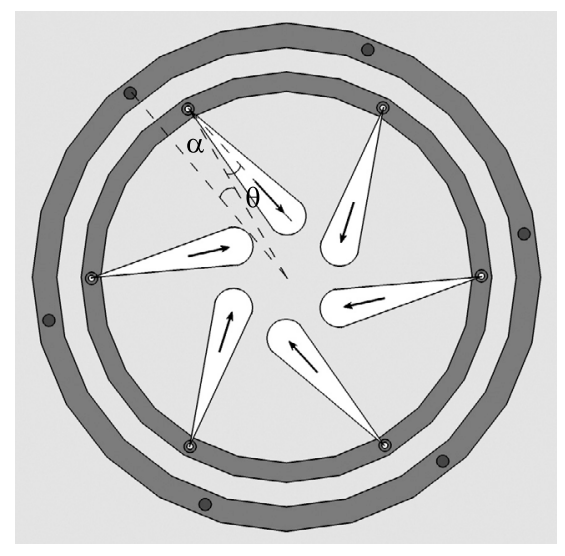

Fig. 1. Top view of the setup. Inner large grey ring: cathode. Outer large grey ring: anode. Small dark-grey circles: return-posts. Small grey-white concentric circles: wires. To close the electric circuit, the wires are connected with the return posts by a bridge electrode (not shown) above the array.

employ a setup based on a modified cylindrical wire array instead, and we explore it numerically to prove the relevance to accretion discs physics.

\section{Numerical code and setup}

GORGON (Chittenden et al. 2004; Ciardi et al. 2007) is a resistive, 3D MHD code based on the Van Leer algorithm. Ions and electrons are treated as a single fluid. However, the energy equations are solved separately. In order to follow the electromagnetic fields, the vector potential $\mathbf{A}$ is diffused and advected. The code includes radiation losses, Ohmic heating, and a "computational vacuum" below a density $\rho_{v a c}=10^{-4} \mathrm{~kg} / \mathrm{m}^{3}$, in which only the wave equation for $\mathbf{A}$ is solved.

In cylindrical wire arrays, the coronal plasma formed around the wires is accelerated towards the axis by the $\mathbf{J} \times \mathbf{B}_{\phi}$ force produced by the global toroidal field around the array (Lebedev 2001). In this work, a modified cylindrical wire array is employed. A cartoon of the setup viewed from above is shown in Figure 1. In order to introduce angular momentum to the system, each wire is coupled to a return-post with a small angular displacement $\alpha$. The return-posts perturb the global magnetic field asymmetrically around each wire so that the plasma streams emanating from the wires are deflected off-axis by an angle $\theta$. The plasma streams miss the axis and interact with each other forming a rotating plasma.

As a reference case for our computational study we took an array with radius $r_{a}=8 \mathrm{~mm}$, composed by 12 copper wires with length $l_{w}=1.4 \mathrm{~mm}$. The angular displacement of the return posts was set to $\sim 2.8$ degrees and the anodecathode gap $d_{A G}$ to $1 \mathrm{~mm}$. We focused on the electric current provided by the MAGPIE generator, which was approximated by $I(t)=I_{0} \cdot \sin ^{2}(\pi t / 2 \tau)$, where $I_{0}=1.4 \times 10^{6} \mathrm{~A}$ is the peak current and $\tau=250 \times 10^{-9} \mathrm{~s}$ is the current peak time. The resolution was $50 \mu \mathrm{m}$ for the reference case and $100 \mu \mathrm{m}$ for the other runs. 


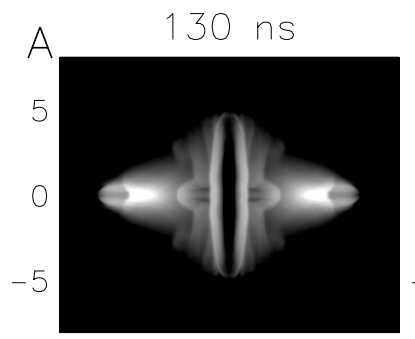

$-5 \quad 0 \quad 5$
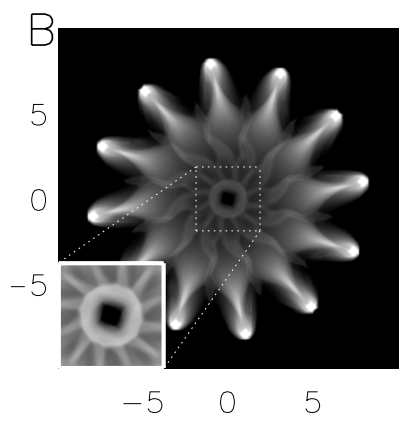

$r(m m)$

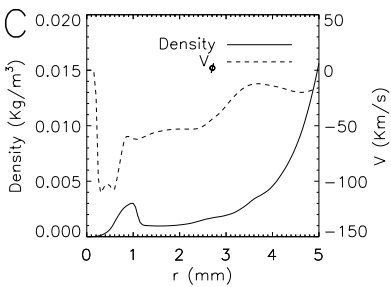

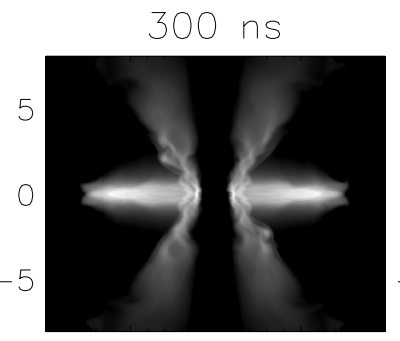

$\begin{array}{lll}-5 & 0 & 5\end{array}$

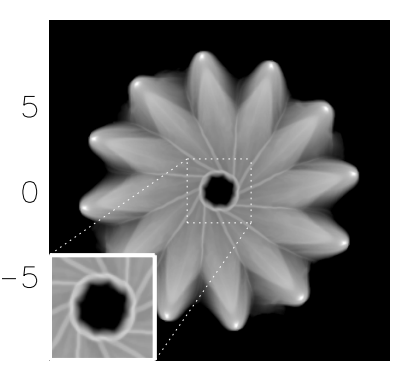

$$
\begin{array}{lll}
-5 & 0 & 5
\end{array}
$$

$r(m m)$

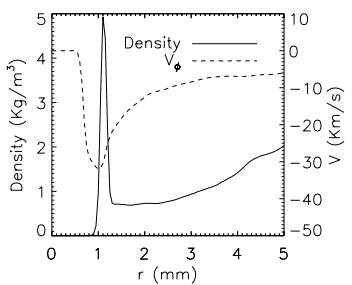

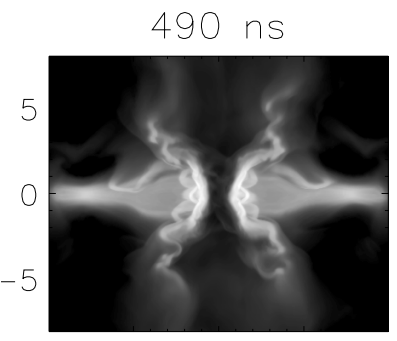

$\begin{array}{lll}-5 & 0 & 5\end{array}$

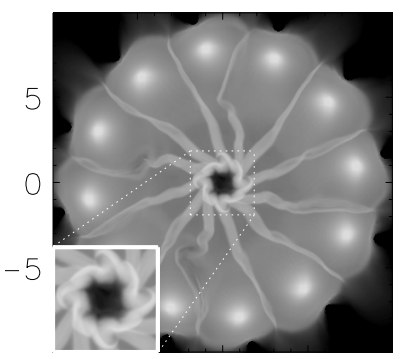

$$
-5 \underset{r(\mathrm{~mm})}{0} 5
$$

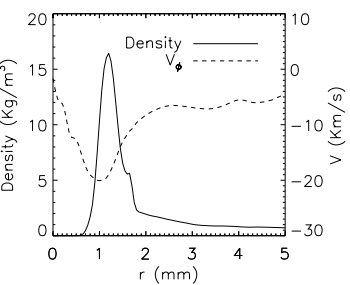

Fig. 2. Time sequence of slices of mass density on the $X Z(\mathbf{A})$ and $X Y(\mathbf{B})$ planes. From left to right: 130, 300 and 490 ns. Only part of the computational domain is shown. The color scale is logarithmic, ranging from $10^{-4} \mathrm{~kg} / \mathrm{m}^{3}$ (black) to variable levels (lighter tones) to highlight structures. The central region is magnified in the insert at the bottom-left corner. $\mathbf{C}$ ): time sequence of average radial profiles of density (solid line) and toroidal velocity (dashed line). Times as above.

\section{Results}

Simulations show that a plasma cylinder or ring is formed, as illustrated by the density cuts in Figures $2 \mathrm{~A}$ and $\mathrm{B}$. We infer from the average radial profiles of density and velocity (Fig. $2 \mathrm{C}$ ) that the ring has radius $\sim 1 \mathrm{~mm}$. The velocity profiles show differential rotation, with a peak velocity of $\sim 35 \mathrm{~km} / \mathrm{s}$ at $250 \mathrm{~ns}$, slowly decreasing in time. The supersonic rotation, with peak Mach number $\sim 7$, can be sustained for $\sim 2-3$ revolutions. The ring radius is determined by the accumulation of angular momentum contrasted by the streams' ram pressure, in 
qualitative agreement with the analysis in Ampleford (2008). Our case, however, is more complex because the plasma is ejected in a couple of thermally driven conical outflows.

Estimates for the dimensionless parameters of the experimental plasma were calculated using local values on the simulated plasma ring and a length scale of $1 \mathrm{~mm}$. Note that computational dimensionless parameters can be different, as dissipative effects are influenced by the resolution employed. The Reynolds $\left(\sim 10^{7}\right)$ and Peclet $\left(\sim 10^{4}\right)$ numbers are $\gg 1$, similarly to astrophysical accretion discs. In contrast, the magnetic Reynolds number $R e_{M}$ is $\leq 1$. However, magnetic fields do not play an important role in the reference case, as $\beta>1$. We expect that the material used for the experiment would significantly alter this result. Indeed, light materials (like aluminium) are less radiatively cooled, yielding lower Mach numbers and higher magnetic Reynolds numbers than heavy materials (like tungsten). Simulations with different materials and parameters showed that it is possible to achieve $R e_{M} \geq 1$.

Despite a smaller $R e_{M}$ than in the astrophysical case, it is useful to study the effects of an external magnetic field on the system for code verification and to assess the feasibility of future experiments. Two configurations were tested: a uniform vertical field, achievable experimentally with coils above and below the electrodes, and a dipolar field in the center of the domain, experimentally achievable with a permanent magnet or a small coil. This latter setup could be relevant to the interaction of an accretion disc with the dipolar field of a central protostar. Our simulations showed that a modest uniform $\mathbf{B}_{\mathbf{Z}}$ of $\sim 1 \mathrm{~T}$ is sufficient to alter the ring radius and symmetry of the system. In particular, stronger fields produce larger, less symmetric rings. Conversely, an experimentally unfeasible dipolar field of $\sim 40 \mathrm{~T}$ (at $1 \mathrm{~mm}$ from the axis) was needed to evidently affect the system.

\section{Conclusions}

In this paper we presented the results of a numerical investigation to test a new experimental configuration aimed at producing rotating plasma flows relevant to astrophysical accretion discs.

We demonstrated that the proposed setup, based on a modified cylindrical wire array, yields a supersonic, differentially rotating plasma flow which is radiatively cooled and displays high Reynolds and Peclet numbers and a plasma $\beta>1$. Although the magnetic Reynolds number was found to be $\leq 1$, it is possible to tune the setup to achieve a $R e_{M} \geq 1$, reaching a regime better related to accretion discs.

A uniform vertical magnetic field of $\sim 1 \mathrm{~T}$ produces experimentally detectable alterations to the system. Implementation of a setup producing similar plasmas to the ones described here is currently under way on the MAGPIE pulsed power facility. Preliminary results confirm the formation of a rotating plasma ring.

The work was supported by EPSRC Grant No. EP/G001324/1 and by NNSA under DOE Cooperative Agreements No. DE-F03-02NA00057 and No. DE-SC-0001063. 


\section{References}

Ampleford, D.J., 2008, PRL, 100, 035001

Balbus, S.A., \& Hawley, J.F., 1991, ApJ, 376, 214

Chittenden, J.P., et al., 2004, Plasma Phys. Control. Fusion, 46, B457

Ciardi, A., et al., 2007, Phys. Plasmas, 14, 056501

Ji, H., 2011, IAU Symp., 274, 18

Lebedev, S.V., et al., 2001, Phys. Plasmas, 8, 3734

Ryutov, D.D., 2011, Ap\&SS, 336, 21

Shakura, N.L., \& Sunyaev, R.A., 1973, A\&A, 24, 337 